\title{
Circuit
}

Musiques contemporaines

\section{Considérations en provenance de Sirius Considerations from Sirius}

\author{
Michel F. Côté
}

Volume 19, numéro 2, 2009

Stockhausen au Québec

URI : https://id.erudit.org/iderudit/037451ar

DOI : https://doi.org/10.7202/037451ar

Aller au sommaire du numéro

Éditeur(s)

Les Presses de l'Université de Montréal

ISSN

1183-1693 (imprimé)

1488-9692 (numérique)

Découvrir la revue

Citer cet article

Côté, M. F. (2009). Considérations en provenance de Sirius. Circuit, 19(2), 57-62. https://doi.org/10.7202/037451ar
Résumé de l'article

Afin de mesurer l'impact de la musique de Stockhausen sur la musique actuelle, ce courant protéiforme de musique improvisée, qui absorbe si facilement des influences tant savantes que populaires, le musicien et compositeur Michel F. Côté se penche sur la question, et s’y greffant les propos d'autres illustres musiciens associés à ce courant tels le saxophoniste Jean Derome et le turntablist Martin Tétreault. 


\section{Enquête}

\section{Considérations en provenance de Sirius ${ }^{1}$}

MICHEL F. CÔTÉ

[NDLR]: En préparant ce numéro, nous voulions mesurer l'impact de la musique de Stockhausen sur la musique actuelle, ce courant protéiforme typiquement (mais pas du tout exclusivement!) québécois de musique improvisée, qui absorbe si facilement des influences tant savantes que populaires. Pour ce faire, nous proposons un témoignage d'un grand acteur de la scène actualiste, Michel F. Côté, qui nous a livré le texte que voici, qui sonde en même temps d'autres illustres musiciens associés à ce courant, tels le saxophoniste Jean Derome et le turntablist Martin Tétreault.

\section{Avertissement céleste tout en S}

On me propose de cerner l'influence potentielle qu'un remarquable compositeur associé à Darmstadt aurait pu avoir sur la musique actuelle - mais, encore une fois, que signifie cette appellation ${ }^{2}$ ? - alors que je n'ai aucune compétence musicologique et très peu de connaissances du corpus musical et des écrits de l'extravagant monsieur de Sirius (Karleinz n'était pas moins excentrique que Sun Ra - fils de Saturne -, ou Satie, ce dernier ayant l'excuse d'avoir été dadaïste...). J'ai tout de même accepté la proposition, par plaisir
1. À propos de la planète Sirius, voir l'article de Bob Gilmore dans ce numéro, p. 42.

2. Insistons pour disqualifier cette dénomination insignifiante qui n'a de vraisemblable que le mot musique. Vous rétorquerez que l'appellation n'est qu'une convention établie dans l'usage, et qu'il ne faut pas faire plus de chichis que nécessaire. Je répondrai que nommer une esthétique n'est pas une chose banale, et que mal la nommer implique mal la comprendre. Je cite deux extraits de Boutès, de Pascal Quignard: "La musique experte en perdition n'a pas besoin de se protéger avec des images ou des propositions, ni de s'abuser avec des hallucinations ou des rêves" et "Les vrais musiciens sont ceux qui lâchent la corde de la langue. Ils quittent une part d'humanité. Ils font le contraire d'Alcibiade à Athènes. " Faites-en ce que vous voudrez, mais comprenez ceci : en art, il est nécessaire de ne pas se faire épingler, nommer, puis classer définitivement. II est nécessaire d'agir de manière imprévisible, et de fuir toute identification. J'insiste, l'appellation musique actuelle est médiocre. Elle est une variation postérieure et réactive à musique contemporaine, une autre appellation maladroite, également court-circuitée lorsque l'esthétique désignée devient prématurément historique. Musique sursitaire aurait été plus joli, quoique aussi inutile. 
de partager un certain enthousiasme pour les travaux de Stockhausen, et par désir d'avouer quelques hypothèses innocentes, au risque de paraître idiot (mais n'est-il pas agréable, voire utile, d'avoir parfois un air d'imbécillité...).

Afin de tout dire sans détour, avançons que ce pionnier de la musique électronique n'a pas eu davantage d'influence que Louis Armstong, Edgard Varèse, Derek Bailey ou Brian Eno - pour n'en nommer que quatre, extraits de l'invraisemblable désordre des mille noms possibles. Stockhausen n'est qu'un parmi une multitude. J'ajoute que l'art musical est protéiforme et ne saurait tolérer une attitude dogmatique, ou une seule manière d'enchaîner les idées. Cette sentence s'applique singulièrement à l'esthétique actualiste. À l'évidence, une idée semblable traversa l'esprit de Stockhausen durant l'époque brève et remarquable pendant laquelle il multiplia les expériences relativistes (cette musique intuitive que d'autres nomment, à tort, abstraction totale).

Il y a plus d'une décennie, en 1996, dans une réponse exhaustive (et contrariée) au volume 6, numéro 2, de la revue Circuit (numéro titré "Musique actuelle?»), afin de préciser qui sont les actualistes, j'écrivais ceci:

Nous sommes, en quelque sorte, les enfants du vinyle. Avec l'éclosion phénoménale du disque microsillon dans les années 1960, une multitude de musiques inouïes devenaient soudainement audibles pour deux générations de jeunes mélomanes et futurs compositeurs. Désormais, dans nos discothèques, les Beatles côtoyaient Art Ensemble of Chicago et Captain Beefheart; Karlheinz Stockhausen se rangeait aux côtés de Soft Machine et d'Erik Satie. Le temps d'une décennie, de 1965 à 1975, l'aventure fut parfaitement exaltante: une quantité phénoménale d'excellentes musiques, tous genres et toutes époques confondus, étaient dorénavant disponibles au Miracle Mart du coin. Musique concrète, gamelan javanais, blues, aucune primauté de l'une sur l'autre, il n'y avait pas ici de bonne ou de mauvaise musique, qu'une infinité de choix et de goûts à développer en toute liberté. S'établissait ainsi, de façon directe et individuelle, une logique de discernement critique, sans aucun dogme académique. La connaissance est cette richesse qui s'acquiert proportionnellement à notre ouverture sur le monde; le hasard et la nécessité, ces outils qui nous permettent de combler notre soif, même dans un Miracle Mart 3 .

D'une manière ou d'une autre, Stockhausen fut l'un des nombreux bénéficiaires de cette manne propagandiste: dès 1970, certains de ses disques, tombés du ciel par hasard dans un improbable grand magasin de Laval (ou d'ailleurs, dans une autre banlieue sans art), se révélèrent une nourriture miraculeuse pour quelques très jeunes mélomanes sagaces.

\section{Verticalité, surf et rebondissement}

Il serait injuste de prétendre que Stockhausen ne fut pas écouté avec plaisir et attention par les actualistes. Mais n'oublions pas que la musique contemporaine n'est qu'une des esthétiques auxquelles les actualistes s'abreuvent.

Nul doute, Stockhausen fut un compositeur spectaculaire. Plus vertical qu'horizontal, tentant sans cesse d'atteindre les étoiles, il aura été prodigue, créant l'un des plus riches catalogues musicaux du xx ${ }^{e}$ siècle.

3. "L'école du pick-up en 2 faces \& 33 points \& 1/3", Esse, $n^{\circ} 30,1996$, p. 2-15. 
Doué d'une vision périphérique exemplaire, doté d'une curiosité hors du commun, il n'a rien voulu manquer et fut remarquable d'efficacité créative. II est amusant d'observer que ses investigations l'ont mené jusqu'à l'antagonisme: de Boulez à Cage, puis inversement, de Cage à Boulez.

Dans le jeu des influences, Karlheinz eut un adversaire redoutable: John Cage. II n'est pas idiot de les comparer. L'exercice est même stimulant: Stockhausen fut un virtuose à l'européenne, sa filiation axiologique n'avait d'égale que l'efficacité d'un savoir-faire prospectif, rationnel (sauf Sirius...) et intelligent; Cage fut un virtuose à l'américaine, son désir naturel de tabula rasa se doubla d'une inclination pour le compromis zen, avec pour résultat cette quête, ce paradoxe: comment faire sans refaire, comment traquer librement l'invention (ou comment cesser d'imposer tout en composant)? L'un surfe sur une longue tradition de rigueur avide, l'autre rebondit dans un monde récent qui tente de réinventer l'univers perceptif.

L'identité d'une composition est pour Boulez et Stockhausen d'une importance primordiale, et elle l'est aussi pour tous les compositeurs issus de la tradition post-Renaissance. Mais l'identité revêt une signification très différente dans les œuvres expérimentales les plus libres, où l'indétermination de l'exécution garantit que deux versions de la même pièce n'auront pratiquement aucun fait musical perceptible en commun.

Dans cet extrait de Experimental Music ${ }^{4}$, Michael Nyman résume efficacement le fossé qui sépare Cage et Stockhausen, en prime il offre un indice sur l'allégeance des actualistes.

4. Experimental Music, Cage et au-delà, Allia, Paris, 2005, p. 31.
Quel est précisément l'impact de Stockhausen sur la musique actuelle? II n'est pas risqué d'affirmer que Cage a établi une filiation plus estimable, mais elle aussi partielle.

\section{Entre Lenny Bruce et W. C. Fields}

Pendant un bref moment, pour son plus grand plaisir, Stockhausen fut la seule marque de commerce semipopulaire à s'exonder du club sélect des compositeurs de Darmstadt: l'équivalent de Coca-Cola pour les boissons gazeuses, toutes proportions gardées. De 1967 à 1972, il fut pop star, ou presque. Soudainement, au juke-box de la musique contemporaine, Karlheinz est alors celui qui affiche le plus de hits; il est inutile d'en faire la liste. Ses disques se vendent; Deutsche Grammophon, le plus important label qui édite alors ses œuvres, va jusqu'à mettre en marché un Festival of Hits (DG 2538 152, 1974); événement inimaginable de nos jours... Entendons-nous, le règne fut volatil et n'a pas provoqué d'hystérie collective, mais à l'époque, il fut suffisamment important pour que de célèbres musiciens situés à des années-lumières, tels que Miles Davis, Frank Zappa, Anthony Braxton, Robert Wyatt, George Russell, Robert Fripp, etc., s'avouent publiquement admirateurs.

Pourquoi la popularité médiatique de notre virtuose sirien s'emballa-t-elle donc? Qu'est-ce qui a pu favoriser un tel engouement? La musique elle-même, évidemment: elle est bonne et le timing historique était tout aussi bon. Mais ce n'est pas suffisant, il y a aussi I'hypothèse que j'offre maintenant - elle sera peut-être reçue avec suspicion - : le $1^{\text {er }}$ juin 1967, un des disques les plus populaires de l'histoire, Sgt. Pepper's Lonely 
Hearts Club Band, huitième disque des Beatles, voit le jour. Au recto de la pochette, on y découvre les visages de plus de soixante-dix personnalités. Tout en haut de cette galerie de célébrités, entre Lenny Bruce et W. C. Fields, on aperçoit un visage sinistre appuyé sur un poignet, il s'agit de Stockhausen. Autour de Karlheinz, il y a Tony Curtis, Carl Gustav Jung, Bob Dylan, Karl Marx, William Burroughs, Edgar Allen Poe, Oscar Wilde, Marilyn Monroe, quelques gourous et Fred Astaire; une belle compagnie ${ }^{5}$. II n'en fallait pas davantage: à la fin de cette décennie, tous les hippies de la planète écoutaient Hymnen, Telemusik et Stimmung...

N'est-il pas symptomatique de constater que la Stockhausen Foundation for Music elle-même, sur son site, accorde une réelle importance à l'anecdote?

\section{Bric-à-brac de petites observations}

1. Dans la liste de ses étudiants relativement célèbres, on trouve quelques noms liés à l'actualité actualiste internationale: Cornelius Cardew, Holger Czuckay, Hugh Davies, Jon Hassell, Moustapha Gerrhard, Irmin Schmidt et La Monte Young. À la suite de cette liste, au Québec, nous pourrions appeler actualistes Raôul Duguay et Walter Boudreau - à l'époque où ceux-ci s'aventuraient au sein de l'Infonie.

2. Concernant les allégeances spirituelles de Stocky (beam me up), il est superflu d'indiquer que

5. [NDLR] Cf. compte-rendu de Réjean Beaucage du livre d'Olivier Julien (dir.) Sgt. Pepper and the Beatles - It Was Forty Years Ago Today (Ashgate Publishing), dans notre dernier numéro. les actualistes ont peu d'affinités avec ces bondieuseries. Philosophiquement et politiquement parlant, Stockhausen n'inspire pas beaucoup. Ce n'est pas négligeable. D’autres compositeurs furent plus pragmatiques, ayant ainsi une influence extra musicale inséparable du corpus qu'ils ont composé. À cet égard, Cage, encore lui, est un exemple de cohérence artistique.

3. Il y a une sensibilité cinématographique associée aux recherches des actualistes. Lors d'une récente discussion, nous avons été plusieurs à relever une sensibilité similaire chez Stockhausen. Contrairement au potentiel cinématographique du répertoire de Ligeti (rentabilisé par l'industrie cinématographique), celui de Stockhausen a été peu exploité (peut-être le compositeur est-il lui-même en cause). II existe au moins une preuve de ce potentiel: les Brothers Quay ont utilisé Zwei Paare, extrait de Freitag aus Licht comme trame sonore d'un court métrage réalisé en 2000, In Absentia. Ce film a remporté de nombreux prix (même ici à Montréal), et le résultat est remarquable. Cette trame musicale aurait pu être réalisée par un actualiste.

4. Stockhausen offre un répertoire particulièrement stoner... De tous les compositeurs de sa génération, il est celui qui procure le plus de satisfaction lorsque l'auditeur altère chimiquement ses capacités perceptives. Les actualistes sont sensibles à ce phénomène... J'en témoigne.

5. Quelques musiciens, actualistes à leur heure - c'est-à-dire capables de franchir les frontières esthétiques avec bonheur, à la fois interprètes, improvisateurs 
et compositeurs (polyvalence caractéristique d'un actualiste) -, ont œuvré pour le maître allemand: les Français Jean-François Jenny-Clark, Michel Portal et Jean-Pierre Drouet (sur Aus den sieben Tagen, en compagnie également de Vinko Globokar).

\section{Space is the Place}

Les actualistes ont certainement maintenu une relation mélomane avec l'œuvre protéiforme de Stockhausen. Ce n'est pas peu, mais aucun autre lien n'est franchement perceptible.

J'ai questionné un échantillon d'actualistes:

Martin Tétreault admet qu'il a utilisé avec efficacité certains vinyles de Stockhausen (il a, encore récemment, utilisé Kurzwellen). Il aura d'abord fallu qu'il écoute cette musique, et sache en apprécier les vertus musicales.

Jean Derome répond que Karlheinz n'a "pas eu d'influence directe sur les actualistes, mais qu'il est tout de même intéressant d'écouter ses pièces de musique intuitive", et que ce répertoire a des similitudes avec certaines de nos préoccupations musicales, "J'y reconnais beaucoup de parentés avec la musique actuelle"; Derome cite alors le vinyle Ceylon/Bird of Passage (Chrysalis Records, 1975). II est probable que ces parentés auxquelles fait référence Derome sont le résultat d'une démarche générique associée à la musique improvisée, plutôt qu'une preuve de filiation à l'héritage de Stochausen.

Un autre me répond joliment que Stockhausen lui était étranger, qu'il n'était qu'un cousin de Raël, un académicien devenu poussiéreux, mais plein de ressources matérielles. Est-il nécessaire de souligner qu'il existe un réflexe antiacadémique latent chez une bonne proportion d'actualistes?

Un quatrième me dit que Aus den sieben Tagen est un repère; que les expériences de musique intuitive effectuées par Stockhausen à la fin des années 1960 furent très stimulantes.

D’autres encore insistent sur l'omniprésence des dispositifs électroniques dans le répertoire de Stockhausen. Gesang der Jünglinge est quelques fois cité comme référence fondatrice.

Stimmung, Spiral et Telemusik ont aussi frappé l'imaginaire de plusieurs des compositeurs-improvisateurs interrogés.

En bref, d'une manière prévisible, le répertoire mentionné est surtout celui des hits de la fin des années 6o, et Stockhausen n'a vraisemblablement pas provoqué d'exclamations délirantes chez mes interlocuteurs.

Je clos avec ce souvenir révélateur, celui d'un disque écouté au printemps de 1972. J'avais alors 14 ans et revenais d'un Miracle Mart avec Hymnen dans mon sac à dos. Sans trop le savoir, je venais de me procurer mon premier disque de musique étrange... Accroché au guidon de mon vélo mustang, je pédalais à toute vitesse, enflammé par l'audition prochaine d'un des disques de ce fameux compositeur qui avait obtenu la cinquième position des figures historiques présentes sur la pochette de Sgt. Pepper's (unique motif de cet achat à l'aveuglette). Le choc fut spectaculaire. J'avais dans les oreilles quelque chose d'inouï: des sons et un espace formel qui m'étaient entièrement étrangers. Instantanément, Stockhausen fut élevé au rang de 
maître iconoclaste. Pour citer Sun Ra, cette nouvelle musique m'informa que there are other worlds (they have not told you of $)^{6}$. Si cette découverte fut saisissante et induisit un bouleversement perceptif, elle ne fut que le premier jalon d'une suite intersidérale de trouvailles qui n'ont cessé depuis lors d'alimenter mon plaisir de mélomane, et mes travaux de compositeur.
Mais tout cela est de la littérature, la musique n'est qu'une question d'oreille. Terminons en affirmant simplement, indubitablement, que Stockhausen en avait de bonnes: il entendit jusqu'à Sirius...

6. En saturnien irréductible, cousin extraterrestre de Karlheinz, Sun Ra a des titres de pièces qui sont parfois des trésors d'imagination. 